\title{
Carnets
}

Revue électronique d'études françaises de l'APEF

Première Série - 1 Numéro Spécial | 2009

Cultures littéraires : nouvelles performances \& développement

\section{Le réseau: une notion en plein essor dans les études littéraires belges}

\section{Ándré Bénit}

\section{(2) OpenEdition}

Journals

Édition électronique

URL : http://journals.openedition.org/carnets/3694

DOI : $10.4000 /$ carnets.3694

ISSN : 1646-7698

Éditeur

APEF

Édition imprimée

Date de publication : 1 juin 2009

Pagination : 139-162

\section{Référence électronique}

Ándré Bénit, «Le réseau: une notion en plein essor dans les études littéraires belges », Carnets [En

ligne], Première Série - 1 Numéro Spécial | 2009, mis en ligne le 16 juin 2018, consulté le 28 avril 2019. URL : http://journals.openedition.org/carnets/3694; DOI : 10.4000/carnets.3694

\section{(c) (i) (3)}

Carnets est mis à disposition selon les termes de la licence Creative Commons - Atribution - Pas d'utilisation commerciale 4.0 International. 


\title{
LE RÉSEAU : UNE NOTION EN PLEIN ESSOR DANS LES ÉTUDES LITTÉRAIRES BELGES
}

\author{
ANDRÉ BÉNIT \\ Universidad Autónoma de Madrid \\ andre.benit@uam.es
}

\begin{abstract}
Résumé
Depuis quelques années, les historiens de la littérature francophone de Belgique s'accordent à signaler que l'utilisation de la notion de réseau littéraire, appelée à compléter la théorie des champs littéraires, peut s'avérer des plus propice à une description plus fine d'un ensemble périphérique comme le sous-champ belge où, en raison d'un faible degré d'institutionnalisation, l'activité littéraire s'organise et se structure selon des modalités propres. Nous nous proposons ici de voir dans quelle mesure quelques études très récentes menées à bien par des chercheurs des Universités de Bruxelles et de Liège permettent de confirmer cette hypothèse et de bousculer quelques-uns des présupposés bien ancrés, telle la logique implacable des piliers et familles.
\end{abstract}

\begin{abstract}
For many years, historians of the francophone literature of Belgium agree to point out that the use of the notion of réseau littéraire (literary network), bound to complete the theory of literary fields, can be more appropriate to a more subtle description of a peripheral whole as the Belgian subfield where, due to a low level o institutionalization, the literary activity is organized and structured according to their own features. At this point, we plan to analyse insofar as some very recent studies done by researchers from the Universities of Brussels and Liege allow to confirm this hypothesis and to shake up some of the established presuppositions as the implacable logics of families and pillars.
\end{abstract}

Mots-clés: réseau, pilier, famille, institution, Belgique.

Keywords: network, pillar, family, institution, Belgium. 
Dans Le dictionnaire du littéraire, Rémy Ponton et Paul Aron indiquent que la sociabilité, "disposition psychologique à se plaire dans la compagnie des autres", est un mécanisme social important dans la vie intellectuelle, et qu'en matière de littérature, celle-ci se manifeste dans des activités comme les académies et les cénacles, les salons et les cafés : "Elle fonde les réseaux grâce auxquels les auteurs se lient avec les autres auteurs et avec ceux qui les lisent, les soutiennent ou les critiquent", offrant ainsi "un élément d'équilibre à l'activité essentiellement solitaire qu'est la création littéraire, et un canal à la diffusion des idées et des modèles. En retour, la littérature est aussi un facteur de sociabilité" (Ponton et Aron, 2004: 573). Plus loin, ils précisent que la sociabilité propre au monde littéraire exerce ses effets sur l'écriture et sur la réception des œuvres :

Au fil de l'histoire, il apparaît que les formes de lien social privilégient certaines formes d'écriture. Ainsi les salons favorisent la poésie de circonstance, les académies l'éloge, les cafés la poésie, alors que les romanciers semblent avoir davantage eu recours aux repas en commun (ibid: 574 ).

C'est dire qu'il existe là "un champ de recherches pour l'étude des réseaux de diffusion des modèles esthétiques en même temps que pour celle des idées" (ibid.: 574).

De leur côté, dans leur Sociologie de la littérature, Aron et Viala dressent un inventaire de ce qu'une étude sociologique du littéraire doit prendre en compte. Ainsi énumèrent-ils les faits et les questions à observer : "de quoi ?" : de quoi parle-t-on, de quels discours, de quels textes... ? ; "pour quoi ?" : s'agissant du littéraire, la question des effets est primordiale ; “à qui ?" : à qui ces effets adviennent-ils ?; et, enfin, "qui ?" : "qui produit ces textes? Ce qui engage bien évidemment l'observation des auteurs, mais aussi celle des différents acteurs et réseaux et instances qui interviennent dans la vue (sic !) littéraire" (Aron et Viala, 2006: 60). Nos auteurs établissent également une liste des différents "objets" spécifiques -au sens épistémologique du terme- à examiner, proposant de les aborder dans l'ordre suivant -tout en signalant qu'il serait légitime de procéder en ordre inverse : 1. le genre ; 2 . les contenus; 3 . les effets ; 4 . la diffusion et la réception; 5 . l'auteur et la production des œuvres ; 6 . les institutions, réseaux et champ ; et ils soulignent, entre autres choses, la nécessité d'examiner avec soin la situation sociale individuelle de l'écrivain autrement dit sa trajectoire : milieu d'origine, formation reçue, professions exercées...- tout comme la situation sociale collective du groupe dont il fait partie.

Considérant à leur tour que "la question du contexte est cruciale pour la compréhension des œuvres" (ibid:: 100), Aron et Viala soulignent la nécessité de prendre en compte les réseaux qui apparaissent dans l'ensemble des pratiques exercées par les 
institutions tant "de la vie littéraire" (académies, cénacles, mécènes, critique, prix,...) que "supralittéraires" (École, édition, ministères de la culture, bibliothèques...) :

Réseaux d'écrivains qui se soutiennent mutuellement et réseaux constitués entre des écrivains, des éditeurs et des critiques (il n'est pas rare, de nos jours, qu'un écrivain soit aussi critique et directeur de collection chez un éditeur) ; parfois aussi réseaux qui unissent des écrivains et des éditeurs et critiques à telle ou telle instance politique ou idéologique (un parti, une faction religieuse...) (ibid.: 83) ;

en effet, "les effets de réseaux constituent souvent un facteur explicatif important de certaines prises de position et de certaines œuvres, comme aussi de certains succès -ou d'échecs" (ibid.: 83).

\section{Le projet CIEL}

Dans "Littérature belge et recherche collective" (2006), Paul Aron, Benoît Denis et Jean-Marie Klinkenberg affirment qu'à "A nouvelles méthodes, nouveaux objets". Ils y relatent, entre autres choses, leur expérience à la tête d'une "Action de Recherche concertée" de la Communauté française de Belgique (CFB) réunissant, au sein d'un Collectif Interuniversitaire d'Étude du Littéraire (CIEL), des équipes des Universités de Liège et de Bruxelles autour d'un projet (2002-2007) qui fut présenté en détail par Bibiane Fréché lors du Forum APEF 2006. Comme l'indique le titre de sa communication: "Les études de littératures francophones et la notion de réseau", les principaux volets de ce projet sont liés à une réflexion globale sur un concept somme toute assez récent -et de plus en plus présentdans le domaine de l'histoire littéraire et plus précisément de la sociologie de la littérature : celui de réseau littéraire.

Comme l'indiquent Aron et Denis dans leur introduction à l'ouvrage édité par Daphné de Marneffe et Benoît Denis (2006), le recours à cette notion émane du constat suivant : les théories sociologiques actuellement disponibles ayant été élaborées dans le cadre des grands ensembles littéraires nationaux fortement institutionnalisés -tel le champ français-, les méthodes et concepts qu'elles utilisent s'appliquent parfois difficilement à un domaine comme celui des lettres belges de langue française pour lequel elles n'ont pas été conçues et dont, en toute logique, elles ne peuvent prendre en compte toutes les spécificités internes ; il importe donc d'analyser dans quelle mesure l'utilisation de cette notion, appelée à compléter la théorie des champs littéraires, peut se révéler propice à la description plus 
fine d'un tel ensemble périphérique où, en raison d'un faible degré d'institutionnalisation ${ }^{1}$, l'activité littéraire s'organise et se structure selon des modalités différentes. Cette hypothèse, Denis l'avait déjà formulée dans une étude intitulée "La littérature francophone de Belgique. Périphérie et autonomie" :

Du point de vue de l'organisation interne du champ, on constatera que la logique des ruptures distinctives et de la succession des écoles n'est guère présente en Belgique et que l'activité littéraire s'y développe davantage à travers la constitution de réseaux de connivences et d'alliances, qui induisent de la sorte une grande plasticité dans la distribution des positions à l'intérieur du sous-champ (Denis, 2005: 181-182)².

Notons avec Fréché que le syntagme "institution faible" -qui "relève" la notion plutôt péjorative (et non théorisée) de "sous-champ (du champ littéraire français séparé de lui par une frontière politique)" avancée par Bourdieu (1985) pour désigner l'espace littéraire francophone belge, et qui traduit la relation d'interdépendance qu'entretiennent en Belgique les espaces littéraire et social (les relations sociales effectives y jouent un rôle déterminant)ne doit guère être compris "comme un déficit d'autonomie (car les valeurs du monde littéraire sont globalement les mêmes qu'en France) mais plutôt comme la coexistence des logiques sociétale et littéraire" (Fréché, 2007: 206) ${ }^{3}$.

\section{La notion de réseau littéraire dans quelques études littéraires belges récentes}

Si l'on consulte les études publiées depuis une bonne vingtaine d'années sur les lettres belges de langue française, on constate que le terme réseau n'y est pas tout neuf. En effet, Quaghebeur l'utilise déjà dans ses célèbres "Balises pour l'histoire de nos lettres" (1982); analysant les différentes attitudes d'écrivains qui refusaient de lire l'inscription de

\footnotetext{
${ }^{1}$ Denis et Klinkenberg présentent une liste des conditions d'existence de la littérature comme fait social autonome: "En résumé, une littérature autonome présente les caractéristiques suivantes: (1) elle fixe ses propres règles et valeurs [...]; (2) elle organise son personnel [...] ; (3) elle organise ses lieux [...] ; (4) elle travaille à l'élaboration d'un discours sur le littéraire et à la constitution d'une hiérarchie des œuvres et des auteurs ; (5) pour ce faire, elle génère ses propres instances de reconnaissance et de consécration [...] ; (6) et surtout, elle élabore sa propre langue : la langue littéraire ou langue d'écriture" (Denis et Klinkenberg 2005: 31).

2 "De fait, la faiblesse du capital symbolique empêche souvent les agents littéraires périphériques de s'organiser en écoles ou en groupes. La Belgique francophone, par exemple, a produit très peu d'écoles littéraires. Tout au plus des écrivains rallient-ils une école parisienne, comme le firent les symbolistes ou les naturalistes. Les grands schèmes d'organisation de la production littéraire française -comme la division en écoles et genres- rendent donc très mal compte de la structuration littéraire des périphéries" (Fréché, 2007: 207).

3 "D'une part, les auteurs belges agissent au niveau local (maisons d'édition, presse spécialisée, prix littéraires, sociétés, académies, bourses,..., belges), où les effets de la pilarisation de la société sont omniprésents. D'autre part, ils se définissent par rapport au champ littéraire français et participent à ses différents aspects. Ils sont donc constamment tiraillés entre des univers antinomiques (d'un côté, les instances politico-culturelles locales, de l'autre, la prétention française à l'universalité). De cette situation schizophrénique résultent des attitudes de dénégation absolue (je suis un écrivain français), de revendication locale (je suis un écrivain belge) ou de compromis" (Fréché, 2007: 206).
} 
I'histoire dans la littérature francophone de Belgique et, partant, de lire l'inscription de cette littérature dans l'histoire, il écrit que

D'aucuns, qui appartiennent plutôt à la littérature officielle, se revendiquèrent comme les tenants d'une littérature française de Belgique tout en se dotant d'une Académie propre et en jouant de réseaux et de modes qui rendaient leur osmose avec Paris plus que jamais impossible (Quaghebeur, 1982: 19).

Si l'on s'en tient à la dernière décennie, il apparaît très clairement que l'historien de la littérature belge qui a le plus exploité cette notion pour traiter de son champ d'étude, n'est autre que Paul Aron. Ainsi, dans la "Postface" ${ }^{4}$ à la deuxième édition des Balises (1998), évoquant la "lutte de succession" qui se produisit à la suite du décès, en 1973, de Roger Bodart -considéré comme le patron du Ministère de la Culture dont il dirigeait le département littéraire-, Aron signale qu'

une nouvelle génération d'écrivains et de critiques, formés vers 1968, souvent dotés d'une forte culture universitaire et marqués par l'évolution des sciences humaines en pleine ébullition, [...] entra en concurrence avec les réseaux d'amitié et d'habitus littéraire des milieux qui géraient les organes de décision (Académie, Ministère, Fonds des lettres....) sous l'ère Bodart (Aron, 1998: 414-415).

Plus loin, insistant sur le fait que, dans le champ belge, les liens entre politique et littérature se nouent d'une manière originale, Aron signale que

le monde littéraire s'inscrit bon gré mal gré dans les réseaux formés par les trois 'piliers' catholique, libéral et socialiste de la vie sociale belge, qui se sont dotés d'organes spécialisés dans les questions syndicales, politiques ou artistiques recrutant leurs animateurs, voire leurs lecteurs, à partir de leur réseau initial. En concurrence pour l'hégémonie sociale, ces trois grandes familles doivent s'investir dans tous les champs spécialisés, en tentant une difficile liaison entre la défense de leurs intérêts propres et le respect des règles du champ, qui conditionne l'efficacité de leur intervention. De là, la surprenante permanence dans le champ littéraire de réseaux formés en dehors de lui. [...]. Ainsi l'interpénétration des milieux politiques et culturels paraît bien constituer un trait qui se maintient à travers toute l'histoire culturelle belge (ibid.: 421-422).

\footnotetext{
${ }^{4}$ Ce texte développe un article publié en 1995 par Paul Aron : “Littérature belge ou littérature de Belgique ?". In: Pierre Bourdieu (dir.). La colère des Belges. Liber, no 21-22, pp. 24-26.
} 
Assez curieusement, dans les articles théoriques inclus dans Littératures belges de langue française. Histoire \& Perspectives (1830-2000), un ouvrage publié en 2000 sous la direction de Christian Berg et de Pierre Halen, c'est à peine s'il y est question de réseau. En effet, seul Halen, dans son chapitre: "Situation d'une littérature francophone: les 'lettres belges'”, signale l'apparition d'initiatives nouvelles qui, contournant autant que possible la référence parisienne, "tablent à la fois sur le contact direct avec les lecteurs et sur des réseaux inter-régionaux, qui n'ont que faire des frontières nationales, ni d'ailleurs des variables linguistiques" (Halen, 2000: 336).

Pour y dépister la notion de réseau littéraire, il faut donc se plonger dans quelquesuns des chapitres panoramiques, tout spécialement ceux rédigés par Marianne Michaux et par Paul Aron. Dans son étude sur "La difficile conquête de l'autonomie", Michaux utilise la notion de réseau littéraire à trois reprises (pp.24, 39, 40), par exemple, lorsqu'elle évoque les différents milieux gravitant autour des Revue trimestrielle et Revue de Belgique, un réseau d'intellectuels et d'écrivains, formé autour de l'Université libre de Bruxelles, de la francmaçonnerie, de la Libre pensée et de la Ligue de l'enseignement et qui proposait "un modèle de société ou plutôt un contre-modèle du monde catholique et doctrinaire qui, de l'enfance (question de l'éducation) à la mort (question des enterrements civils), prenait en charge tous ses membres au long de leur vie" (Michaux, 2000: 39). Dans son étude sur "Des années folles à la drôle de guerre", Aron insiste, lui, sur le rôle des réseaux qui, après la Première Guerre, se créent au niveau international et grâce auxquels des écrivains comme Tousseul, Plisnier, Nougé, Seuphor ou de Ghelderode se feront entendre à Paris, voire dans l'Europe entière. C'est ainsi, dit-il, que Tousseul réalise de manière singulière une carrière, discrète sans doute mais qui "doit autant aux réseaux de la littérature populiste et prolétarienne qu'à ceux du régionalisme" (Aron, 2000: 150).

D'autres brèves références à la notion de réseau apparaissent sporadiquement dans le même ouvrage: sous les plumes d'Angelet (2000: 80-81), de Quaghebeur (2000:187-188), de Berg (2000: 389-390), ainsi que de Durand et Wilkin, pour qui il n'y a

pas non plus de livre ni de revue symboliquement efficaces si ces véhicules matériels ne sont pas à leur tour relayés par des instances de réception, recension, consécration, elles-mêmes inscrites dans le réseau immatériel qu'aménage au sein du microcosme culturel le jeu des places et des positions qu'y occupent, en connivence autant qu'en concurrence, les différents acteurs, lieux et milieux de sociabilité lettrée qui en sont constitutifs (Durand et Wilkin, 2000: 439).

Dans l'Histoire de la littérature belge francophone 1830-2000 publiée en 2003 sous la direction de Jean-Pierre Bertrand, Michel Biron, Benoît Denis et Rainier Grutman, la notion 
de réseau n'est guère davantage à l'honneur. Elle y est utilisée par deux chercheurs, québécois et français, à savoir Michel Biron (à propos de Camille Lemonnier, p.144) et Hélène Védrine, laquelle traite du réseau littéraire créé par Félicien Rops, qu'elle définit comme "un artiste de la liaison, du passage, de l'entre-deux, dont la fonction est à saisir dans le mouvement qui s'établit entre la France et la Belgique, entre réalisme et symbolisme, entre littérature et peinture" (Védrine, 2003: 92). Bien entendu, le concept est exploité par Aron qui, dans son chapitre sur "Les écrivains belges et l'occupation", évoque notamment quelques-uns des réseaux d'amitié qui jouèrent alors un rôle non négligeable (Aron, 2003: 406). La notion apparaît également (et timidement) dans les chapitres qu'Aurore Boraczek consacre à l'art social en Belgique à la fin du XIX ${ }^{e}$ siècle (Boraczek, 2003: 177), et dans celui de Jacques Dubois sur la création de la CFB et de la Promotion des lettres où s'est développé un réseau, informel mais effectif, au gré des relations plus ou moins étroites que les écrivains entretiennent avec ce service (Dubois, 2003: 507-508).

En réalité, c'est dans $L a$ littérature belge. Précis d'histoire sociale, un ouvrage publié en 2005, que la notion de réseau se déploie, semble-t-il, pour la première fois dans toute son ampleur et sa richesse. Evoquant les mutations que l'historiographie connaît depuis le début des années septante, outre l'organisation des jeunes universitaires en équipes, centres de recherches et réseaux -éventuellement universitaires (comme le CIEL)-, ainsi que la mise sur pied de réseaux de lecteurs de la CFB, Denis et Klinkenberg (2005: 88) pointent comme facteur important du renouvellement de l'historiographie littéraire belge l'apport de deux modèles, la sociologie des champs de Bourdieu et de l'institution littéraire de Dubois, dont les concepts théoriques (centre vs périphérie, autonomie, insécurité linguistique, réseau, etc.) sont largement utilisés dans leur ouvrage. A titre d'exemple, parmi bien d'autres (pp.133, 139, 158, 175-176, 178, 195), retenons l'importance, bien connue, de la revue dans la "Renaissance des lettres belges" au cours des années 1880, un organe que nos auteurs définissent comme un lieu de publication mais aussi comme un espace de sociabilité :

elle rassemble des écrivains et leur donne donc une masse critique ; elle met sur pied des réseaux et permet aux partisans de telle ou telle thèse de se compter ; elle permet aux groupes de se différencier les uns des autres ; elle permet que la création s'accompagne d'un discours d'escorte critique (Denis et Klinkenberg, 2005: 127) ;

d'ailleurs, l'expression Jeune Belgique ne désigne-t-elle pas à la fois le titre de la revue et le groupe des écrivains qu'elle a permis de réunir? 


\section{La portée théorique et pratique de la notion de réseau littéraire}

Notre intention n'est pas ici de tenter de discuter la pertinence d'un terme comme celui de réseau, encore moins de proposer une nouvelle définition de cette notion polysémique et fuyante. Les études présentées dans le volume Les réseaux littéraires (2006) publié par Denis et de Marneffe indiquent bien, selon nous, que la rigueur scientifique dont ils font preuve n'empêche nullement les chercheurs d'adapter au mieux cette notion à leur objet d'étude. II s'agira donc ici de rappeler brièvement quelques éléments concernant ladite notion et d'observer le profit que les travaux sur la littérature francophone de Belgique peuvent tirer concrètement de son utilisation.

Situé au point d'interaction de l'histoire culturelle et de la sociologie de la littérature, le concept de réseau que Dozo et Fréché définissent comme "l'ensemble complexe des relations qui s'établissent, au sein d'un espace culturel et social donné, entre divers acteurs, groupes ou institutions, relations qui assurent en outre l'unité et la cohérence de cet espace" (Dozo et Fréché, 2006: 86), se présente comme un outil performant pour examiner la manière dont "un ensemble littéraire s'organise et se structure, aussi bien dans la multiplicité de ses relations internes (la vie littéraire au sens strict) que dans son rapport à d'autres secteurs d'activités (les autres arts, les sphères intellectuelle ou médiatique, les divisions sociopolitiques, etc.)" ; pour "restituer le contexte de la production littéraire [...] comme celui de sa réception à différentes époques [...]" (Aron et al., 2006: 95-97) ; et, partant, pour "rendre compte des relations et de la structure du personnel littéraire belge francophone -à savoir l'ensemble des participants à cette vie littéraire (auteurs, éditeurs, responsables d'institutions culturelles, etc.)" (Fréché, op. cit.: 207).

En effet, appliquée adéquatement, cette "théorie du réseau" -qui ne prétend nullement concurrencer celle des champs (beaucoup plus élaborée et dont l'efficacité n'est plus à démontrer lorsqu'il s'agit de décrire les positions dominantes), mais qui peut la compléter (Lacroix, 2006; Sapiro, 2006)- semble davantage appropriée à l'analyse des ensembles dominés (littératures périphériques, régionalistes, ou paralittératures), à la description des "institutions littéraires faibles" telle la belge, à l'intérieur de laquelle la difficulté qu'éprouvent les littérateurs à produire du capital symbolique en incite un grand nombre à miser sur leur capital relationnel, défini par Aron et Denis comme "la capacité plus ou moins grande que possède un agent d'utiliser ses liens (d'amitié, de connivence, de proximité idéologique, etc.) en vue de produire certains effets" (Aron et Denis, 2006: 16).

Dans son "premier bilan" à l'analyse des réseaux littéraires, Viala (2006: 266) nous met en garde contre quelques-unes des tentations qui guettent les chercheurs et les critiques, comme celle de rebaptiser "réseau", par souci de modernité, des choses étudiées auparavant sous d'autres noms. D'où l'importance: premièrement, de définir des critères 
stricts permettant de délimiter et d'analyser ce que sont réellement les réseaux littéraires, car "ces derniers ne sont pas ceux que tel ou tel critique peut dessiner, selon ses curiosités et selon des liens thématiques qu'il peut établir entre des textes, mais bien des réseaux ayant effectivement impliqué des personnes" (Viala, 2006: 269) ; ensuite, de veiller à caractériser leur structure (très variable) selon divers paramètres (de leur dimension -notamment temporelle- jusqu'au degré de centralité et d'intermédiarité de certains réseaux ou transversalité par rapport à d'autres...) ; enfin, d'être conscient que les réseaux sont de puissance et de solidité différentes selon le niveau où ils interviennent (ainsi, lorsqu'on examine un réseau de la superstructure sociale (tel un réseau littéraire), il est indispensable de le connecter à des réseaux infrastructurels ( $p$. ex: le substrat socio-économique) et structurels (les réseaux qui ordonnent les structures de la vie sociale, telles les familles (catholique, socialiste et libérale) qui, en Belgique, gèrent la vie politique, la protection sociale et l'enseignement) ${ }^{5}$.

Dans son "Avant-propos" à "La vie et l'œuvre" ? Recherches sur le biographique (2008), Jérôme Meizoz insiste sur l'importance non seulement de "saisir un auteur dans les 'réseaux' qui sont les siens", mais aussi de "comprendre les logiques de sa 'trajectoire' dans le champ littéraire" (Meizoz, 2008: 5). Parmi les critères essentiels pour mener à bien une analyse réticulaire cohérente, Viala et Klinkenberg soulignent eux aussi la nécessité d'octroyer une place prépondérante aux écrivains, en associant le concept de réseau à celui de trajectoire d'auteur ("à chacune des phases de la trajectoire littéraire d'un auteur correspond l'intervention spécifique d'une instance constituée par l'action de réseaux spécifiques" (Klinkenberg, 2006: 71)), et plus particulièrement aux moments névralgiques de la biographie d'écrivain: émergence (personnelle et publique), légitimation et reconnaissance, et enfin consécration (pour reprendre les trois phases de la trajectoire d'un écrivain telle que la décrit Dubois dans L'institution de la littérature (cf. Bertrand, 2005) ; phases précédées de celle de la formation et des débuts dans l'écriture, selon Klinkenberg pour qui la trajectoire implique à la fois une carrière et des filières :

une carrière, c'est-à-dire le passage par une série de positions, mais aussi des filières, notion qui renvoie à l'existence d'instances extérieures, et notamment d'instances de légitimation. L'étude de la trajectoire postule ainsi celle de la sociabilité

\footnotetext{
5 "La société belge est fortement marquée par l'institutionnalisation tous azimuts de deux lignes de partage idéologiques. La première, qui oppose depuis 1830 les catholiques aux non-catholiques, a engendré deux mondes idéologiques (deux 'piliers', en néedandais zuilen) ayant chacun ses propres organisations (politiques, syndicales, culturelles, etc.) ainsi que ses propres institutions (réseaux scolaires, soins de santé, etc.). Ce phénomène est appelé 'pilarisation' (verzuiling). Le second clivage, socio-économique et opposant dès 1880 les socialistes aux libéraux conservateurs, a provoqué la scission du 'pilier' laïque et continue de fragiliser le 'pilier' catholique. Aujourd'hui, la plupart des Belges naissent vivent et meurent au sein d'un de ces trois États dans l'État, et ce souvent à leur insu. En effet, les 'piliers' (appelés aussi les 'familles'), si opaques et envahissants soient-ils, finissent par passer inaperçus, en ce qu'ils produisent un nombre élevé de conditions 'indispensables' mais impensées des pratiques sociales" (Dirkx, 1995: 8).
} 
subie, qui rend compte des positions de départ, autant que celle de la sociabilité choisie, qui rend compte des prises de position (Klinkenberg, ibid:: 75).

A partir de là, commente Viala, il devient possible de "discerner quels autres paramètres, quels autres acteurs de la vie littéraire (éditeurs, critiques, mécènes, etc.) et donc quels autres réseaux sont à prendre en compte et permettent ainsi de construire des contextes" (Viala, op. cit.: 270). Ainsi s'agira-t-il par exemple, dans le cas de l'émergence de “La 'nouvelle génération' romancière face à ses réseaux (1997-2001)" étudiée par JeanPierre Bertrand et Anthony Glinoer, de divulguer l'importance des réseaux médiatiques et commerciaux. Nul doute dès lors que l'analyse des réseaux -qui, de par son apport en matière d'observation et de construction des contextes, peut aider à réévaluer certains des regroupements opérés par l'histoire et la critique littéraires- constitue un réel enrichissement pour l'étude du littéraire.

Dans la "conclusion" de leur étude "Réseaux et bases de données" dans laquelle ils considèrent que, contrairement à la notion de champ qui permet de décrire à la perfection un espace littéraire homogène et structuré (comme l'est le champ français), la notion de réseau, souple et ouverte, convient mieux dans le cas des pratiques littéraires faiblement autonomisées (comme l'est le "sous-champ" belge où le tissu relationnel entre le personnel littéraire et politique est particulièrement serré), Dozo et Fréché signalaient que les différents usages de la base de données CIEL seraient l'objet des premières thèses de doctorat exploitant la méthode d'étude réticulaire, un outil novateur pour les études littéraires belges.

\section{Quelques études récentes}

La première thèse de doctorat, élaborée sous la direction de Paul Aron et soutenue à l'Université libre de Bruxelles $^{6}$ en avril 2006, fut précisément celle de Bibiane Fréché. Comme l'indique son titre: Entre rupture et continuité. Le champ littéraire belge après la Seconde Guerre mondiale (3 septembre 1944 - 8 octobre 1960), cette thèse (inédite) propose une analyse approfondie du champ littéraire belge du second après-guerre. Après avoir retracé les conditions littéraires et culturelles durant la Deuxième Guerre (Première partie: "L’héritage de la guerre"), Fréché étudie l'épuration du champ littéraire, à savoir la réorganisation de celui-ci, par des instances qui lui sont extérieures, avant d'analyser les rapports entre les écrivains et les institutions publiques et littéraires (Deuxième partie : "La réorganisation du champ littéraire") et de s'atteler à la description du champ littéraire de ces quelque quinze années : elle y décrit la position et les prises de positions des différents

\footnotetext{
${ }^{6}$ Voir Catalogue des thèses électroniques de l'ULB.
} 
agents en présence pour y dévoiler l'image d'une institution littéraire faible, incapable de faire émerger un nombre conséquent des auteurs de l'époque (Troisième partie : "Un champ en simulacre"). Dans son article "Pouvoir, littérature et réseaux en Belgique francophone : Roger Bodart (1910-1973)"7, Fréché nous en fournit un échantillon fort intéressant : après le rappel de quelques-unes des caractéristiques générales qui différencient les champs belge et français, elle étudie le "système Bodart" et analyse en détail le rôle institutionnel déterminant joué, après 1945, par celui qu'elle nomme "l'ami précieux" (Fréché, 2008: 62) ; dans ses conclusions, Fréché signale à ce propos que cette figure emblématique de la "vie littéraire belge"8 de l'après deuxième guerre, qui contribua à faire émerger un réseau d'écrivains particulièrement bien connectés à l'époque avec le champ du pouvoir, ne représentait certes qu'un simple nœud de relations de ce réseau, mais un nœud efficace et incontournable; par ailleurs, elle invite les futurs chercheurs à se pencher sur le rôle actuellement joué en faveur de la littérature belge par d'autres écrivains hauts fonctionnaires comme Jean-Luc Outers et Marc Quaghebeur, respectivement directeur du Service de la promotion des lettres et commissaire du livre de la CFB, et ce "afin de mesurer plus largement l'efficacité du 'capital relationnel' des écrivains, dans les relations qu'ils nouent avec des agents du champ du pouvoir" (ibid:: 68).

Deux autres thèses ont été soutenues en 2007 à l'Université de Liège, celle de Daphné de Marneffe, dirigée par Jean-Pierre Bertrand, et celle de Björn-Olav Dozo, dirigée par Jean-Marie Klinkenberg ${ }^{9}$.

Dans sa thèse intitulée Entre modernisme et avant-garde. Le réseau des revues littéraires de l'immédiat après-guerre en Belgique (1919-1922), de Marneffe analyse, dans une perspective d'histoire et de sociologie de la littérature, l'ensemble des revues littéraires "modernistes et d'avant-garde" parues en Belgique au cours de ces quatre années, l'exemplification portant essentiellement sur les corpus des revues francophones d'Anvers (La Drogue, Lumière, Ça Ira) et de Bruxelles (L’Art libre, Haro, Au Volant, Demain littéraire et social, Le Geste, Signaux de France et de Belgique, La Lanterne sourde, 7 Arts, Sélection).

Dans la première partie, consacrée à l'explicitation des "Choix théoriques et méthodologiques", c'est en terme de réseau qu'est appréhendé l'ensemble de ces revues littéraires bruxelloises et anversoises de l'immédiat après-guerre, un réseau de revues que de Marneffe définit en fonction de quatre caractéristiques (un espace sans clôture, hétérogène, régi par une logique de solidarité et déployé dans la durée) et qui se développe

\footnotetext{
${ }^{7}$ Voir également son analyse sur "Le réseau des trois mousquetaires" (Fréché, 2007: 211-213).

${ }^{8}$ Fréché précise que, les concepts et méthodes de la théorie des champs de Bourdieu ne pouvant être appliqués tels quels au corpus littéraire belge en raison de ses particularités intrinsèques, elle préfère aux termes de "champ littéraire belge" ceux de "vie littéraire belge", "institution littéraire belge", "sous-champ littéraire belge" (Fréché, 2008: 55).

${ }^{9}$ Voir Réseau des Bibliothèques de I'Université de Liège, BICTEL/e - Ulg, Serveur institutionnel des thèses de doctorat.
} 
dans une double dimension : humaine (la revue comme lieu de sociabilité, comme milieu) et textuelle (la revue comme lieu de publication, comme instance éditoriale).

La deuxième partie : "Entre restauration de l'avant-guerre' et engagements d'avantgarde'. Reconstitution progressive de l'espace des revues à l'Armistice", traite principalement de la situation de soumission, durant cette période de transition, des enjeux esthétiques aux questions politiques ainsi que des prises de position (plus idéologiques qu'esthétiques) des revues dans le débat enflammé sur la reconstruction (dans tous les sens du terme) du pays, conçue en terme de restauration par le pôle conservateur -qui se fit le champion de l'épuration- et en terme de renouvellement et création à neuf (sur tous les plans : social, politique, architectural, urbanistique, artistique, culturel, littéraire) par le pôle moderniste et d'avant-garde -alors soudé pour les besoins de la cause ${ }^{10}$.

Dans la troisième partie, de Marneffe explore le réseau intertextuel et interpersonnel des revues littéraires modernistes et d'avant-garde à travers l'examen minutieux des espaces où se déploie l'“action d'art" desdites revues (centres d'art, galeries et lieux d'expositions, tribunes médiatiques, maisons d'édition) ainsi que des milieux imbriqués dans les corpus bruxellois et anversois. Ceci lui permet de montrer que le réseau des revues modernistes et d'avant-garde, tant au niveau des ancrages personnels qu'au niveau des relations interpersonnelles et intertextuelles, doit être situé "dans un continuum plus large, celui du réseau global des revues de l'époque, toutes tendances confondues" (de Marneffe, 2007: 301). En effet, la forte cohésion du réseau interpersonnel et intertextuel des revues étudiées n'exclut pas l'existence de liens, certes discrets mais constants, avec les revues et les milieux plus conservateurs de l'espace littéraire, tant sur le plan de l'imbrication entre les milieux que sur celui des appuis concrets sollicités par les revues modernistes et d'avantgarde pour assurer leurs conditions d'existence ; ainsi, dans la société belge où la logique de la "pilarisation" est souvent décrite comme implacablement assujettissante, ces revues se révèlent être "des lieux d'intersection entre les différents milieux, des zones de débat, où peuvent se définir des positions intermédiaires"; les agents qui les occupent ont beau témoigner d'une recherche d'autonomie et d'indépendance, "la fragilité de leur position se traduit dans un grand nombre de cas par l'adhésion à d'autres types de valeurs, menant parfois à la collaboration pendant la seconde guerre mondiale"; une telle fragilité se perçoit également "au niveau matériel, ces revues 'progressistes', hors piliers, éprouvant toutes des difficultés très concrètes à se maintenir" ; c'est dire qu'ici le réseau ne se résume pas

\footnotetext{
10 "Faisant front contre un ennemi commun, les revues modernistes et d'avant-garde se rejoignent dans la promotion d'une vision du monde alternative à celle proposée par le pouvoir en place. Pour l'heure, elles n'ont pas encore défini de programme esthétique suffisamment précis pour s'opposer les unes aux autres sur ce point. Cette conjoncture particulière explique en partie l'importance de la logique de solidarité qui régit le 'réseau des revues' : toutes ont intérêt à développer des échanges interpersonnels et de se faire écho mutuellement dans leurs colonnes afin de faire exister un espace de parole et de réception favorable à leurs idées" (de Marneffe, 2007: 298).
} 
à un ensemble de relations interpersonnelles ou de citations intertextuelles : celles-ci sont les indices d'un type de fonctionnement propre à cet espace des revues, régi par une logique de solidarité, dictée en partie par la conjoncture politique et matérielle de l'immédiat après-guerre (ibid.: 301-302).

La quatrième et dernière partie : "Prolongements et conclusion", offre un retour sur le modèle réticulaire, à travers les problématiques

- de l'inscription du réseau des revues littéraires modernistes et avant-gardistes dans la durée -et ce à travers l'examen de deux questions, (1) celle des "filiations" entre revues (à travers plusieurs cas de figure: la revendication de ces liens dans les propos liminaires des revues elles-mêmes, l'établissement de ces liens grâce à divers témoignages enregistrés par l'histoire littéraire (même marginale), ou par déduction personnelle du chercheur) ; (2) celle du travail historiographique (car les revues s'efforcèrent très rapidement de poser les jalons de leur histoire) :

la revendication 'textuelle' d'une filiation peut aussi être lue comme une question d'étiquette et de transfert symbolique de 'label', une question d'enjeu historiographique [le travail d'inscription dans l'histoire n'est-il pas un des enjeux internes à l'espace des revues ?]. C'est assez clair dans les 'préfaces tardives' qui accompagnent les éditions reprint (ibid.: 288) ;

- du rapport de la littérature avec les autres champs :

La notion d'objet transactionnel' peut être utile ici pour désigner la revue littéraire comme lieu d'une articulation dynamique entre les champs. [...] Les revues littéraires, par leur plasticité et par le fait qu'elles déploient leur action en combinant différents moyens textuels et médiatiques, apparaissent comme un lieu privilégié de transactions [...] entre les différentes sphères de production (abordées sous l'angle du rapport au public), entre la presse et la littérature et, plus globalement, entre les différents champs culturels, artistiques et social (ibid.: 291-292).

- et, enfin, de l'inscription du réseau des revues littéraires modernistes et avantgardistes dans un espace à extension variable (en raison du caractère translinguistique et cosmopolite dudit réseau), ce qui conduit de Marneffe à la conclusion -provisoire- que ces revues investissent massivement dans une stratégie d'ouverture internationale ou cosmopolite, "manière d'échapper à la fois à la dimension 'belgo-belge' des débats politiques, de répondre à l'impératif a-linguistique 
du champ artistique et de résister à la force d'attraction du champ littéraire français" (ibid:: 294-295).

Et de conclure en soulignant, notamment, la prégnance de l'effet de réseau reliant les différentes revues littéraires modernistes et d'avant-garde au cours de cette période, certes brève ${ }^{11}$ mais ô combien décisive pour le futur du royaume de Belgique.

Dans l'introduction de sa thèse intitulée Mesures de l'écrivain. Étude socio-statistique du sous-champ littéraire belge francophone de l'entre-deux-guerres, Dozo indique d'une part que son objectif est celui de mobiliser une démarche quantitative afin d'étudier la littérature belge, en particulier son personnel littéraire qu'il définit comme "l'ensemble des personnes participant de près ou de loin à la vie littéraire, qu'il s'agisse d'écrivains [...], de critiques, d'éditeurs, de personnes travaillant pour des instances médiatiques, pour des instances décisionnelles en matière de littérature, etc." (Dozo, 2007: 9) ; d'autre part, que ses recherches, qui s'inscrivent dans la sociologie des champs de Bourdieu, mobilisent deux approches complémentaires, à savoir l'histoire sociale de la littérature (tradition de recherche en littérature initiée à Liège par Jacques Dubois) et la sociologie quantitative, une synthèse personnelle de ces deux approches ${ }^{12}$ s'avérant nécessaire pour analyser le fonctionnement du sous-champ littéraire francophone belge de l'entre-deux-guerres.

La première partie comprend une introduction et trois chapitres. Dans le premier, Dozo précise d'une part qu'il mobilise la méthode prosopographique ${ }^{13}$ et explicite d'autre part son outil (la base de données du $\mathrm{CIEL}^{14}$ ) et son corpus (les auteurs belges ayant publié une œuvre littéraire durant l'entre-deux-guerres). Les chapitres deux et trois offrent une archéologie de deux méthodes d'analyse quantitative utilisées par la suite: l'analyse factorielle des correspondances multiples ${ }^{15}$ et l'analyse structurale des relations sociales ou analyse structurale des réseaux sociaux ${ }^{16}$, chacun des deux chapitres revenant sur les théories principales qui mobilisent ces méthodes en sciences sociales (la théorie des

\footnotetext{
${ }^{11}$ Neutralisés par l'effet de réseau, les effets de champ resurgissent dès le moment où se dessinent des lignes de force : "On ne s'étonnera pas que les revues surréalistes se positionnent, à partir de 1924, par démarcage vis-àvis des 'modernistes' (comme Correspondance se moquant de 7 Arts)" (de Marneffe, 2007: 299).

${ }^{12}$ Concernant la synthèse de l'analyse prosopographique, combinée à la théorie des champs, et de l'analyse structurale des relations sociales effectives, ainsi que pour une réflexion théorique et méthodologique sur deux méthodes et sur deux outils dont dispose le chercheur en sociologie de la littérature, voir Dozo 2008a.

${ }^{13}$ La prosopographie consiste "en la définition d'une population à partir d'un ou de plusieurs critères, puis à l'établissement à son propos d'un questionnaire biographique dont les diverses variables serviront à la décrire dans sa dynamique sociale, privée, publique, voire culturelle, idéologique ou politique, selon la population et le questionnaire retenus" (Dozo, 2008a: 2 ).

${ }^{14}$ La collecte de données entreprise par le CIEL a permis de rassembler des données relationnelles effectives de deux types : les données d'appartenance (à des institutions, des groupes, des manifestes, des jurys, etc.) et les données de relations directes (lettres et correspondances, préfaces, etc.).

15 Outil statistique de présentation et d'analyse de tableaux de données, l'analyse factorielle des correspondances est l'outil privilégié de la théorie des champs, l'objectif étant de classer un nombre d'individus en fonction de variables qui les décrivent.

${ }^{16}$ Outil d'analyse mathématique, l'analyse structurale des relations sociales est l'instrument privilégié de la théorie sociologique des réseaux.
} 
champs pour l'analyse factorielle, la théorie de l'action rationnelle pour les relations sociales ${ }^{17}$ ). Dozo propose ensuite une synthèse des apports de ces deux méthodes utilisées dans le cadre de la théorie des champs, moyennant une discussion de certaines implications de cette synthèse, et illustre chaque méthode : la première, par une analyse factorielle du personnel de l'Académie royale de Langue et Littérature françaises de Belgique (ARLLF), ce qui lui permet notamment de noter un basculement dans le recrutement social de ce personnel; la seconde, par une analyse relationnelle des préfaciers et des auteurs d'ouvrages parus dans l'entre-deux-guerres, laquelle lui permet de détecter la forte structuration du réseau catholique en Belgique francophone et d'introduire une catégorie de littérateurs qui retiendra ultérieurement son attention : les "animateurs de la vie littéraire".

La deuxième partie comprend, elle aussi, trois chapitres. Dans le premier, Dozo problématise son corpus autour de la notion d'autonomie du littéraire et d'indépendance de la littérature belge par rapport au champ français ; s'appuyant sur la suggestion de Denis -à savoir l'existence d'un champ littéraire belge à considérer comme un sous-champ du champ français, plus ou moins indépendant en fonction des époques-, il s'interroge sur la capacité de cet espace littéraire faiblement institutionnalisé que constitue la Belgique francophone de l'entre-deux-guerres à produire sa propre norme et à se doter de ses propres règles de fonctionnement, l'hypothèse étant que la logique réticulaire joue alors un rôle capital dans le processus de reconnaissance des écrivains (Dozo, 2008a: 17). Pour vérifier celle-ci -et, partant, afin de reconstituer le réseau de relations interpersonnelles des écrivains francophones belges d'alors (c'est-à-dire leur participation aux lieux de sociabilité belges francophones) et de comprendre quel type de capital symbolique est reconnu aux agents les plus impliqués dans le sous-champ littéraire belge- Dozo construit, dans le deuxième chapitre, les variables pertinentes pour l'analyse, à savoir, outre les indicateurs économiques et culturels (c'est-à-dire les capitaux économique et culturel, traditionnels dans la théorie des champs), le capital spécifique au champ littéraire, ou capital symbolique qu'il subdivise en capital symbolique belge, celui acquis et utilisé en Belgique ${ }^{18}$, et capital symbolique français, celui acquis en France mais exploité en Belgique, l'enjeu de ce découpage étant de montrer la double stratégie qui s'offre aux littérateurs ; ce capital symbolique dont les indicateurs sont

\footnotetext{
17 Degenne et Forsé (1994: 11) résument en trois propositions la théorie moderne du choix rationnel :

a) les individus agissent de façon à atteindre les objectifs qu'ils se sont fixés en fonction de leurs préférences ;

b) des contraintes interfèrent sur les actions en influençant les probabilités que certaines préférences puissent être atteintes ; ces contraintes sont institutionnelles ou structurales ;

c) compte tenu de ces contraintes, les individus choisissent les actions qui leur permettent de se rapprocher le plus possible de leurs préférences et donc des fins qu'ils poursuivent (voir Dozo, 2008a: 11-12).

18 "La plupart de ceux qui animent la vie littéraire belge francophone n'ont aucun nom en France: ils publient chez des éditeurs belges, participent à des revues belges et côtoient des écrivains belges. Cette absence de relation avec le centre parisien ne les empêche pas d'exister, voire d'acquérir une notoriété importante au sein du sous-champ littéraire belge. / L'important pour exister dans le petit monde littéraire n'est pas nécessairement de publier beaucoup de livres et de rencontrer un large succès. Le modèle des champs de production de biens symboliques de Bourdieu le décrit bien : c'est aux yeux des pairs qu'il faut exister" (Dozo, 2008b: 184).
} 
les prix littéraires, les éditeurs et les genres, il le corrèle avec le capital relationne/ ${ }^{19}$ défini comme "l'ensemble des liens directs ou indirects que l'agent capitalise grâce à sa position structurale dans son réseau, mesurés grâce aux outils de l'analyse structurale des réseaux sociaux" (ibid: 17). Enfin, Dozo introduit le correctif générationnel pour la classe d'âge de chaque agent de son corpus. Dans le troisième chapitre, il s'interroge, moyennant plusieurs analyses factorielles, sur les corrélations existant entre les différents indicateurs ${ }^{20}$ ainsi que sur les effets de celles-ci :

- l'analyse montre que le capital symbolique belge est relativement indépendant du capital symbolique français, en particulier pour la génération littéraire qui, après la Première Guerre, est confrontée à la reconfiguration des stratégies littéraires belges francophones, lesquelles passent grosso modo d'une logique centrifuge (affirmation nationaliste belge) à une logique centripète (fascination pour le centre parisien) (Denis et Klinkenberg, 2005). Ce constat traduit une certaine autonomie du souschamp littéraire belge francophone par rapport au champ français, et partant la possibilité d'une reconnaissance spécifique par ce sous-champ.

En effet, cette génération de la transition, "qui doit assurer la conversion du mythe nordique en autre chose" (Dozo, 2007), est composée de groupes qui, tels les signataires du Manifeste du Lundi emmenés par Franz Hellens et Robert Poulet, le PEN Club ou les Scriptores Catholici, s'efforcent d'ouvrir de nouveaux horizons dans l'espace littéraire belge francophone (respectivement, le rapport -certes ambigu- à la France, car désormais l'identité n'est plus nationale mais linguistique ; l'internationalisation de la littérature ; et l'émergence d'un "art pour Dieu"). Bien entendu, cette nouvelle génération ne jouit guère d'une reconnaissance parisienne comparable à celle des Jeune Belgique, mais elle partage avec celle-ci un capital symbolique belge fort (plus élevé que celui d'origine française), témoignant ainsi qu'il est possible de déterminer une forme de capital propre au sous-champ belge, et que ce capital symbolique belge dépend de la capacité de ces agents à élaborer des enjeux propres à ce sous-champ (lesquels, moyennant quelques arrangements, peuvent être décrits comme potentiellement valorisables dans le champ français).

- la double opposition -(1) capital économique faible et capital culturel faible vs capital économique fort et capital culturel fort ; (2) générations consacrées vs générations émergentes)- non seulement marque les corrélations existant entre les générations

\footnotetext{
19 "La faiblesse institutionnelle que l'on constate dans le champ littéraire belge francophone a permis l'émergence d'écrivains grâce à des stratégies moins traditionnelles que celles mises en œuvre dans le champ littéraire français. En particulier, la variable que Bourdieu nomme le 'capital social' prend une dimension remarquablement importante dans le champ littéraire francophone belge" (Dozo, 2008b: 184).

${ }^{20}$ Chacun de ces indicateurs est le produit synthétique d'indices variés (comme l'origine sociale, la profession, la profession des parents, la formation (les études), l'état civil (situation maritale pour les revenus de l'épouse), l'évolution socio-professionnelle, la participation à des groupes littéraires, à des institutions, à des revues, les hobbies, etc.).
} 
socio-littéraires et ces capitaux économique et culturel, mais elle confirme aussi la modification qui s'opère à l'époque dans le profil social des agents -modification déjà constatée avec le personnel littéraire de l'ARLLF. En ce qui concerne la "génération tampon" -celle de la transition entre les phases centrifuge et centripète-, Dozo signale que sa physionomie hésite entre le prolongement des profils sociaux des générations antérieures (qui disposaient en général de capitaux culturels et économiques bien supérieurs) et la prise en compte de la rupture socio-politique qui s'est opérée après la Grande Guerre : le bouleversement -causé par cette rupture- de l'espace des possibles de ces littérateurs contraint ceux-ci à renégocier leur place dans le champ littéraire et, de ce fait, à modifier la configuration sociale du champ littéraire lui-même. C'est en effet à partir de cette génération que les profils sociaux des agents littéraires belges francophones connaissent une profonde évolution: le profil du grand bourgeois juriste cultivé bascule vers celui, sociologiquement plus médian, du petitbourgeois enseignant ou fonctionnaire. Dozo ajoute que cette évolution des profils sociaux est corrélée à celle des prises de position esthétiques et de la conception de la littérature défendue par ces agents, une évolution qui justifie la périodisation de l'histoire de la littérature belge en trois phases (centrifuge, centripète et dialectique). Cette génération a donc un profil hybride : car, si plusieurs de ses membres -tel Hellens $^{21}$ - offrent une physionomie proche de celle des membres des générations antérieures, la plupart, qui ont un capital économique et une formation plutôt faibles, présentent des traits propres aux membres des générations suivantes : ils font partie "de la masse des littérateurs anonymes que secrète tout ensemble littéraire : ceux que l'institution de l'époque ne met pas en avant, ceux qui ne bénéficient que très peu d'une reconnaissance contemporaine à leur activité" (Dozo 2007). Néanmoins, de cette troupe de méconnus émergent aujourd'hui ceux que les historiens de la littérature belge ont longtemps "oubliés" : les surréalistes.

Comment expliquer cette singularité ? En ce qui concerne le capital relationnel, bien que les surréalistes participent à de nombreux lieux de sociabilité (manifestes et groupes), ils ne sont que très peu (voire nullement) connectés au reste du réseau formé par les liens entre tous les littérateurs de l'époque ; en effet, le surréalisme est un mouvement isolé qui a sa propre forme de sociabilité, laquelle repose sur des affinités électives : il faut être choisi pour participer ; cette sélection drastique des liens entretenus ne favorise point une participation active aux institutions de la vie

\footnotetext{
${ }^{21}$ Cette génération partage encore avec les générations précédentes un capital culturel fort : "On parle souvent de Franz Hellens comme de l'héritier direct, mais tardif et isolé, de la génération symboliste, en évoquant en particulier sa formation (même collège -Sainte-Barbe à Gand- et même type d'études -le droit- que les plus éminents représentants de cette génération -Maeterlinck par exemple). [...] il n'est pas le seul et [...] d'autres parmi sa génération, comme Alex Pasquier ou Pierre Nothomb, avaient des profils très comparables" (Dozo, 2007).
} 
littéraire. En ce qui concerne le capital symbolique, le constat est proche: ne bénéficiant guère de la légitimité conférée par l'institution littéraire contemporaine, les surréalistes ne peuvent alors prétendre à une reconnaissance de leurs pratiques. Cette double exclusion (relationnelle et symbolique) les place donc dans la masse des anonymes des lettres belges.

- l'analyse souligne la corrélation existant alors entre les capitaux symboliques français et surtout belge et le capital relationnel. Ainsi, dans la Belgique francophone de l'entre-deux-guerres, les littérateurs dotés d'un important capital symbolique belge bénéficient souvent d'un capital relationnel fort tandis que les littérateurs mal ou peu introduits dans la vie littéraire n'ont que peu de capital symbolique -ce qui n'a rien de surprenant. En revanche, "les capitaux symboliques belge et français moyens sont très proches du capital relationnel fort", ce qui, selon Dozo, est très révélateur des modalités du fonctionnement du sous-champ littéraire belge francophone:

il n'est pas nécessaire d'avoir une reconnaissance énorme pour être en mesure de mobiliser un capital relationnel fort. Le lien entre capital symbolique fort et capital relationnel fort est nécessaire mais pas suffisant : le premier entraîne nécessairement le second, mais l'inverse n'est pas vrai (Dozo, 2007).

On le voit, ce constat confirme lui aussi l'hypothèse de départ selon laquelle le souschamp littéraire belge de l'entre-deux-guerres produit, en marge du champ français, sa propre forme de norme sociale, laquelle est fondée sur la logique du réseau (Dozo, 2008a: 18).

Aux deux questions qu'il se posait au départ : (1) Peut-on parler de sous-champ de la littérature belge francophone? (2) Cet espace est-il assez autonome pour exister comme champ d'une pratique spécifique qui dicte ses propres lois, et assez indépendant du champ français pour ne pas y être assimilé ?, Dozo (2007) répond donc résolument par l'affirmative. L'autonomie du sous-champ littéraire est en effet confirmée par une série d'indices sociolittéraires (existence de maisons d'édition spécifiques, en particulier celles issues des revues littéraires, présence de lieux de sociabilité dédiés à la littérature et émergence d'instances de consécration propres au sous-champ) ; pour ce qui est de l'indépendance par rapport au champ français, la réponse doit être plus nuancée : si le capital symbolique acquis en France joue un rôle primordial pour la reconnaissance en Belgique, le sous-champ belge a développé un capital national spécifique qui peut être mesuré sous la forme d'un capital symbolique classique acquis en Belgique et/ou grâce au capital relationnel d'un agent. La corrélation étroite entre les valeurs de ces deux capitaux permet de confirmer que le mode 
de fonctionnement privilégié au sein du sous-champ littéraire belge francophone de l'entredeux-guerres est bien le réseau.

Dans ses conclusions générales, Dozo signale qu'un tel constat a des implications importantes pour la recherche future sur le sous-champ littéraire belge : il peut en effet servir de fondement pour élaborer de nouvelles hypothèses et ouvrir des pistes de recherche. Et de proposer une voie à explorer, celle de la catégorie des "animateurs de la vie littéraire", une catégorie particulière d'écrivains, dotés d'un capital relationnel fort et dont le rôle, dans un espace relationnellement aussi structuré, doit être abordé avec des outils adéquats,

à même de rendre raison des multiples facettes du statut de ces gestionnaires de l'espace littéraire: préfacier régulier, directeur de revue, secrétaire de groupe littéraire, voire haut fonctionnaire du service public impliqué dans les instances décisionnelles, toutes ces fonctions peuvent être réparties ou cumulées. Si l'analyse des relations sociales permet de baliser le corpus et de mettre en évidence les agents clés, elle doit être complétée par une étude qualitative de ces pratiques, notamment une recontextualisation socio-historique centrée sur certains agents et une explicitation des enjeux attachés à leur position. II faudra par exemple s'interroger sur le fait que par leur position même, ces agents ont tout à gagner en donnant de la visibilité aux débats sur la littérature belge : ceux-ci sont en effet bénéfiques à leur position, étant donné que cette dernière est spécifique au sous-champ littéraire belge. Dans leur chef, ne pas faire exister ce débat revient donc à remettre en question sa propre existence dans le sous-champ littéraire. À partir de là, on peut légitimement se demander dans quelle mesure ces animateurs de la vie littéraire ne sont pas également les premiers producteurs de discours identitaire en littérature (Dozo, 2007).

Les propositions de Fréché et de Dozo se recoupent donc étroitement.

Dans son article "Structure de l'espace relationnel des auteurs francophones belges de l'entre-deux-guerres", Dozo explore l'activité préfacière des membres du personnel littéraire francophone belge à cette époque; il s'y centre sur la préface comme forme de sociabilité en littérature, moyen d'existence dans le champ littéraire et lien fort. Car, si la préface -dont les usages et utilités sont multiples- opère d'habitude un transfert de capital symbolique du préfacier ( $p$. ex. un écrivain consacré) vers le préfacé ( $p$. ex. un écrivain débutant), il n'est pas rare qu'elle bénéficie aussi, voire d'abord, à qui la rédige : “C'est alors l'acte de préfacer qui construit le préfacier en tant qu'auteur dans le sens où il tire son existence dans le champ littéraire de sa capacité à produire des préfaces pour d'autres" (Dozo, 2008b: 189). D'ailleurs, certains écrivains ne sont-ils pas davantage reconnus pour leur rôle d'animateur de la vie littéraire que pour leur œuvre littéraire? 
Dans cette étude où il tente de déceler les logiques qui sous-tendent les réseaux de préfaces entre les écrivains francophones de Belgique au cours de l'entre-deux-guerres ${ }^{22}$, Dozo analyse le rôle de quelques-uns de ces "animateurs de la vie littéraire" à partir de l'examen de leur capital relationnel pour constater, notamment, l'importance -attendue- de l'effet générationnel dans l'activité de préfacier ("Généralement, ceux qui préfacent le plus sont souvent les plus âgés"), mais aussi la prégnance de cette activité dans la structuration des différents réseaux ("Par leur abondante production de préfaces, ils structurent chaque réseau autour d'eux" (ibid: 198)) $)^{23}$. Par ailleurs, il identifie trois réseaux principaux, constitués davantage sur le critère "tendance politique et idéologique" que sur l'effet générationnel ou la composante "mouvement littéraire". Le premier réseau repéré étant à dominante catholique, la tentation était forte d'examiner les autres réseaux comme étant les représentants des deux autres piliers traditionnels en Belgique francophone : le socialiste et le libéral. Toutefois, signale Dozo, ce serait travestir la réalité que de présenter les trois réseaux comme la personnification pure et simple en littérature des trois grandes familles politiques. En effet, s'ils ont comme plus petit dénominateur commun leur ancrage laïque par opposition au réseau catholique-, les deux réseaux en question présentent "le même type de configuration hybride" et ne peuvent être clairement identifiés à l'un desdits piliers : au sein du réseau qui se caractérise par une "forte composante progressiste", les éléments libéraux côtoient les militants de gauche; dans l'autre réseau, il n'existe pas non plus une unique tendance politique : ainsi "l'opposition entre socialistes et libéraux dans les organes de presse et en littérature n'est pas aussi tranchée que ne le laisserait supposer le système des piliers politiques" (ibid:: 196-197). Pour confirmer cette porosité, à l'époque, des réseaux de gauche et de droite, Dozo signale que ces deux tendances ont une même origine (leur opposition au conservatisme catholique), encore assez proche dans le temps (elle remonte à la fin du XIX ${ }^{e}$ siècle) et qui leur permet de coopérer.

Pour résumer l'interprétation possible de cette division en trois réseaux, il faut souligner les éléments suivants : la présence en littérature d'un pilier catholique est indéniable. Opposé à lui, on trouve les laïques qui se structurent en deux piliers,

\footnotetext{
22 "Sont-elles de nature littéraire sur le mode des logiques d'inclusion et d'exclusion qui prévalent pour la constitution des groupes ou des écoles littéraires ? Sont-elles de nature générationnelle ? Ou encore de nature extralittéraire, dues par exemple aux clivages politiques ?" (Dozo, 2008b: 192).

${ }^{23}$ Pensons par exemple à Maurice Gauchez qui eut une production de poète, mais dont l'histoire littéraire se souvient essentiellement pour ses activités d'animateur et d'éditeur de revue ; il fut un des principaux rouages du cercle bruxellois constitué autour de la revue La Renaissance d'Occident et qui rassemblait Michel de Ghelderode, Camille Poupeye, Max Elskamp et Max Deauville: "[Gauchez] usait dans les lettres de rappel à l'ordre adressées aux titulaires des différentes rubriques de la revue, d'un ton extrêmement décidé, qui ne souffrait pas la discussion. Son activité de directeur et d'éditeur l'a donc amené à construire autour de lui un réseau serré dont on trouve la trace dans son activité de préfacier" (Dozo, 2008b: 199). Selon Dozo, bien que n'étant pas d'obédience catholique, ce groupe littéraire peut être considéré "comme un 'tampon' entre la composante principale du réseau catholique, autour de Henri Carton de Wiart, et le groupe autour de Léon Debatty, lui aussi à tendance catholique" (Dozo, 2008b: 195).
} 
socialistes et libéraux. Néanmoins, l'opposition entre socialistes et libéraux est moins structurante dans le champ littéraire que l'opposition entre laïques et catholiques et des collaborations littéraires entre socialistes et libéraux sont monnaie courante (ibid: 197).

\section{En guise de conclusion}

Certes, la recension des liens n'étant pas complète (pour atteindre l'exhaustivité, il faudrait collecter l'ensemble des références d'ouvrages préfacés), cette configuration ne doit pas être considérée comme statique et définitive. Toutefois, insiste Dozo, il convient de "remarquer la valeur heuristique de ce découpage qui met au jour certaines problématiques particulières du champ belge, notamment la perméabilité par rapport aux clivages politiques" (ibid:: 197-198). Par ailleurs, il faut tenir compte du fait que l'acte de préfacer n'est qu'une des stratégies (certes importante) permettant aux littérateurs de s'imposer comme "animateurs de la vie littéraire" et donc d'exercer une influence dans le champ littéraire belge francophone de l'époque. A cet égard, Dozo précise que la confrontation des données relationnelles effectives et des données caractéristiques des agents offre un large programme de recherche, "surtout si l'on se fonde sur les lieux de sociabilité des écrivains, comme les académies, les sociétés ou les groupes littéraires, voire, à d'autres époques, les cénacles ou les salons" (ibid:: 200).

Dans ses conclusions, Dozo souligne une fois encore la nécessité d'approfondir l'examen des différentes pratiques des "animateurs de la vie littéraire" et en particulier du rôle hautement stratégique joué par quelques personnalités institutionnellement puissantes au sein du sous-champ belge; en effet, de par la configuration spécifique de cet espace caractérisé "par l'absence d'institutions centralisées légitimantes fortes", ces notables "structurent le champ littéraire autour d'[eux] et construisent leur légitimité puis l'accordent aux auteurs de leur entourage grâce à un tissu relationnel dense"24. Selon Dozo, c'est grâce à ce type d'agents "que se manifeste et se matérialise l'efficace de l'illusio spécifique partagée par les auteurs belges francophones et donc que l'institution littéraire belge peut exister à côté des logiques du champ français" (ibid.: 201).

\footnotetext{
24 "Cette légitimité se fonde non pas sur une œuvre préalable, reconnue et admirée par ceux qui cherchent à s'imposer dans le champ, mais plutôt sur une reconnaissance mutuelle et un investissement humain souvent très concret, au service de ce qu'ils considèrent tous comme la Littérature" (Dozo, 2008b: 201).
} 


\section{Bibliographie}

ANGELET, Christian (2000). "Vers la reconnaissance internationale". In: Christian Berg, Pierre Halen (dir.). Littératures belges de langue française. Histoire \& Perspectives (1830-2000). Bruxelles: Le Cri, pp. 57-111.

ARON, Paul (1998). "Postface". In: Marc Quaghebeur. Balises pour l'histoire des lettres belges de langue française. Bruxelles: Labor, Espace Nord, pp. 405-423.

ARON, Paul (2000). "Des années folles à la drôle de guerre". In: Christian Berg, Pierre Halen (dir.). Littératures belges de langue française. Histoire \& Perspectives (1830-2000). Bruxelles: Le Cri, pp. 113-173.

ARON, Paul (2003). "1942. Pierre Peyel remporte le concours littéraire du Soir. Les écrivains belges et l'occupation : entre engagement et indifférence". In: Jean-Pierre Bertrand, Michel Biron, Benoît Denis, Rainier Grutman (dir.). Histoire de la littérature belge francophone 1830-2000. Paris: Fayard, pp. 401-410.

ARON, Paul et DENIS, Benoît (2006). "Introduction. Réseaux et institution faible". In: Daphné de Marneffe, Benoît Denis (éd.). Les réseaux littéraires. Bruxelles: Le Cri - CIEL - ULB - Ulg, pp. 7-18.

ARon, Paul et VIALA, Alain (2006). Sociologie de la littérature. Paris: Presses Universitaires de France, Coll. Que sais-je?

ARON, Paul, DENIS, Benoît et KLINKENBERG, Jean-Marie (2006). "Littérature belge et recherche collective". In: Textyles, no 29, pp. 90-97.

BERG, Christian (2000). "La Belgique romane et sa Flandre". In: Christian Berg, Pierre Halen (dir.). Littératures belges de langue française. Histoire \& Perspectives (1830-2000). Bruxelles: Le Cri, pp. 389-412.

BeRTRAND, Jean-Pierre (2005). "Préface". In: Jacques Dubois. L'institution de la littérature. Bruxelles: Labor, Espace Nord / Références, pp. 7-15.

BERTRAND, Jean-Pierre et GLINOER, Anthony (2006). "La 'nouvelle génération' romancière face à ses réseaux (1997-2001)”. In: Daphné de Marneffe, Benoît Denis (éd.). Les réseaux littéraires. Bruxelles: Le Cri - CIEL - ULB - Ulg, pp. 249-262.

BerTRAND, Jean-Pierre, BIRON, Michel, DENIS, Benoît et GRUTMAN, Rainier (dir.) (2003). Histoire de la littérature belge francophone 1830-2000. Paris: Fayard.

BIRON, Michel (2003). "27 mai 1883. Un banquet de réparation est organisé en l'honneur de Camille Lemonnier. L'autonomie nouvelle de la littérature". In: Jean-Pierre Bertrand, Michel Biron, Benoît Denis, Rainier Grutman (dir.). Histoire de la littérature belge francophone 1830-2000. Paris: Fayard, pp. 139-149.

BORACZEK, Aurore (2003). "1891. Verhaeren célèbre la création de la section d'art du Parti ouvrier belge. Rêver et renverser : symbolisme et socialisme belges". In: Jean-Pierre Bertrand, Michel Biron, Benoît Denis, Rainier Grutman (dir.). Histoire de la littérature belge francophone 1830-2000. Paris: Fayard, pp. 171-181.

BOURDIEU, Pierre (1985). "Existe-t-il une littérature belge ? Limites d'un champ et frontières politiques". In: Etudes de lettres, no 4, pp. 3-6.

de MARNEFFE, Daphné (2007). Entre modernisme et avant-garde. Le réseau des revues littéraires de l'immédiat après-guerre en Belgique (1919-1922). Université de Liège. <URL: http://bictel.ulg.ac.be/ETD-db/collection/available/ULgetd-09292007-212823/>.

de MARNEFFE, Daphné et DENIS, Benoît (éd.) (2006). Les réseaux littéraires. Bruxelles: Le Cri - CIEL - ULB - Ulg.

DEgENNE, Alain et FORSÉ, Michel (1994). Les réseaux sociaux. Paris: Armand Collin.

DENIS, Benoît (2005). "La littérature francophone de Belgique. Périphérie et autonomie". In: Jacques Dubois, Pascal Durand, Yves Winkin (dir.). Le symbolique et le social. La réception internationale de la pensée de Pierre Bourdieu. Liège: Editions de l'Université de Liège, pp. 175-184.

DeNIS, Benoît et KLINKENBERG, Jean-Marie (2005). La littérature belge. Précis d'histoire sociale, 
Labor, Espace Nord / Références, №211.

DIRKX, Paul (1995). "Un autre nationalisme”. In: Pierre Bourdieu (dir.). La colère des Belges. Liber, no 21-22, pp. 6-8.

Dozo, Björn-Olav (2007). Mesures de l'écrivain. Étude socio-statistique du sous-champ littéraire belge francophone de l'entre-deux-guerres en Belgique francophone. Université de Liège. <URL: http://bictel.ulg.ac.be/ETD-db/collection/available/ULgetd-09292007-174518/>.

Dozo, Björn-Olav (2008a). "Données biographiques et données relationnelles. Notes théoriques pour une utilisation complémentaire des outils quantitatifs". In: COnTEXTES, no 3. <URL: http://contextes.revues.org/document1933.html>.

Dozo, Björn-Olav (2008b). "Structure de l'espace relationnel des auteurs francophones belges de l'entre-deux-guerres". In: Marie-Pier Luneau, Josée Vincent (dir.). La Fabrication de l'auteur. Québec: Nota Bene, pp. 183-203.

Dozo, Björn-Olav et FrECHE, Bibiane (2006). "Réseaux et bases de données". In: Daphné de Marneffe, Benoît Denis (éd.). Les réseaux littéraires. Bruxelles: Le Cri - CIEL - ULB - Ulg, pp. 86108.

DuBoIs, Jacques (2003). "1981. Création de la Communauté française de Belgique et de la Promotion des lettres. L'institution de la littérature". In: Jean-Pierre Bertrand, Michel Biron, Benoît Denis, Rainier Grutman (dir.). Histoire de la littérature belge francophone 1830-2000. Paris: Fayard, pp. 499-511.

DuBoIs, Jacques (2005). L'institution de la littérature. Essai. Nouvelle édition. Bruxelles: Labor, Espace Nord / Références ( $1^{\mathrm{e}}$ éd. 1978).

DURAND, Pascal et WINKIN, Yves (2000). "L'infrastructure éditoriale". In: Christian Berg, Pierre Halen (dir.). Littératures belges de langue française. Histoire \& Perspectives (1830-2000). Bruxelles: Le Cri, pp. 439-462.

FRECHE, Bibiane (2006). Entre rupture et continuité. Le champ littéraire belge après la Seconde Guerre mondiale (3 septembre 1944 - 8 octobre 1960). Université libre de Bruxelles. <URL: http://theses.ulb.ac.be/ETD-db/collection/available/ULBetd-04242006-102814/>.

FRECHE, Bibiane (2007). "Les études de littératures francophones et la notion de réseau". In: Ana Paula Coutinho Mendes, Maria de Fátima Outeirinho, Maria Hermínia Amado Laurel, Rosa Bizarro (coord.). Espaces de la Francophonie en débat. Forum APEF 2006. Faculdade de Letras, Universidade do Porto, pp. 203-214.

FRECHE, Bibiane (2008). "Pouvoir, littérature et réseaux en Belgique francophone : Roger Bodart (1910-1973)". In: Christine Le Quellec Cottier, Daniel Maggetti (éd.). Écrire en francophonie : une prise de pouvoir ?. Etudes de Lettres, ํㅡㄴ 279, pp. 55-70.

HALEN, Pierre (2000). "Situation d'une littérature francophone : les 'lettres belges'”. In: Christian Berg, Pierre Halen (dir.). Littératures belges de langue française. Histoire \& Perspectives (1830-2000). Bruxelles: Le Cri, pp. 321-339.

KLINKENBERG, Jean-Marie (2004). "Réseau" : In: Paul Aron, Denis Saint-Jacques, Alain Viala (dir.). Le dictionnaire du littéraire. Paris: PUF, Quadrige, p. 538.

KLINKENBERG, Jean-Marie (2006). "Réseaux et trajectoires". In: Daphné de Marneffe, Benoît Denis (éd.). Les réseaux littéraires. Bruxelles: Le Cri - CIEL - ULB - Ulg, pp. 71-85.

LACROIX, Michel (2006). "Ponts, triades, trous et le Tiers inclus. Le cas des relations entre Léo-Paul Desrosiers et les éditions Gallimard". In: Daphné de Marneffe, Benoît Denis (éd.). Les réseaux littéraires. Bruxelles: Le Cri - CIEL - ULB - Ulg, pp. 201-224.

MEIzOZ, Jérôme (2008). "Avant-propos”. In: Philippe Kaenel, Jérôme Meizoz, François Rosset, Nelly Valsangiacomo (éd.). "La vie et l'œuvre" ? Recherches sur le biographique. Université de Lausanne, pp. 3-8.

MICHAUX, Marianne (2000). "La difficile conquête de l'autonomie". In: Christian Berg, Pierre Halen (dir.). Littératures belges de langue française. Histoire \& Perspectives (1830-2000). Bruxelles: Le Cri, pp. 17-56. 
PONTON, Rémy et ARON, Paul (2004). "Sociabilité littéraire". In: Paul Aron, Denis Saint-Jacques, Alain Viala (dir.). Le dictionnaire du littéraire. Paris: PUF, Quadrige, pp. 573-574.

QUAGHEBEUR, Marc (1982). "Balises pour l'histoire de nos lettres". Alphabet des lettres belges de langue française. Bruxelles: Association pour la promotion des Lettres belges de langue française, pp. 9-202.

QUAGHEBEUR, Marc (2000). "De l'Ambiguïté à l'Ouvert ? Soixante ans de littérature belge (19401999)". In: Christian Berg, Pierre Halen (dir.). Littératures belges de langue française. Histoire \& Perspectives (1830-2000). Bruxelles: Le Cri, pp. 175-269.

SAPIRO, Gisèle (2006). "Réseaux, institution(s) et champ". In: Daphné de Marneffe, Benoît Denis (éd.). Les réseaux littéraires. Bruxelles: Le Cri - CIEL - ULB - Ulg, pp. 44-59.

VEDRINE, Hélène (2003). "3 février 1856. Félicien Rops fait paraître le premier numéro de l'hebdomadaire Uylenspiegel, journal des ébats artistiques et littéraires. Rops ou la politique de l'entre-deux". In: Jean-Pierre Bertrand, Michel Biron, Benoît Denis, Rainier Grutman (dir.). Histoire de la littérature belge francophone 1830-2000. Paris: Fayard, pp. 85-93.

VIALA, Alain (2006). "Pour un premier bilan”. In: Daphné de Marneffe, Benoît Denis (éd.). Les réseaux littéraires. Bruxelles: Le Cri - CIEL - ULB - Ulg, pp. 263-280. 